not possible to decide which of the mechanisms was responsible as the exact age of the lesions was not known and sequential specimens were not obtained from the same patient.

When antigen is injected into the vitreous body of a rabbit's eye an inflammatory response occurs in the uvea after about a week, when circulating antibodies appear (Silverstein et al., 1961). Episcleritis is associated with arthritis which is produced by injecting rats with bacteria in adjuvants (Waksman and Bullington, 1956), and the complication is also well known in patients with rheumatoid arthritis (Boyle and Buchanan, 1971). In our patients petechiae, which presumably contained meningococcal antigen, were often seen in the conjunctivae and sclerae at the time of admission. About six days later episcleritis appeared, which may have been the result of a reaction between this depot of antigen and antibodies which were then in production (Artenstein et al., 1971).

The incidence of proteinuria at the time of the complications did not differ significantly from the uncomplicated group. Thus we have no evidence of immune complex nephritis. Either proteinuria was coincidental and due to another cause, such as schistosomiasis, or there was slight transient renal damage related to the preceding infection in both groups.

The arthritis, cutaneous vasculitis, and eye lesions appeared three to five days after the start of therapy, when the patients were recovering from meningococcal infection. At that stage antibiotics had obviously been successful, as all blood cultures except one were sterile and no organisms could be found or cultured from the lesions. Thus we have little evidence that active and persistent infection was the cause of these complications. Penicillin allergy is not a likely explanation as the allergic complications were equally common in the penicillin and chloramphenicol groups during trials of these drugs and were well known before the days of penicillin.

Allergy to meningococcal antigen seems the most likely explanation. In general the patients who developed the lesions suffered severe systemic infection characterized by profuse petechial haemorrhages, joint pains, positive blood culture, and sometimes hypotension. They were also likely to have meningcoccal antigen in their blood (Greenwood et al., 1971). The complications occurred at a time when antibodies were being produced. The histological changes in joints and skin were in keeping with immune complex disease.

One odd feature of this group was the high incidence of people over the age of 30 , an age when meningococcal meningitis is uncommon in the meningitis belt (Lapeyssonie, 1963). Either these patients were unusually susceptible to the infection or, as Morse et al. (1971) suggested, the allergic reactions are a feature of an anamnestic response to past infection. This is not likely as patients who have high haemagglutinating antibody titres on admission have less severe disease, and in such cases meningococci or circulating antigens are rarely detected (B. M. Greenwood and $\mathrm{H}$. C. Whittle, unpublished). It is known that patients with chronic meningococcaemia who also develop arthritis and skin lesions are unable to agglutinate the infecting organism (Benoit, 1963). Perhaps our group of patients had a defective or slow antibody response which allowed a more severe infection. Large amounts of antigen would then circulate in the blood and be deposited in synovia, skin, and the eye. Later, when antibodies are produced, antigen is still in excess and damaging complexes are formed either directly at these sites (Arthus-type situation) or in the blood, from which they are deposited in the vessels of these structures (serum sickness situation).

\section{References}

Abu-Nassar, H., Hill, N., Fred, H. L., and Yow, E. M. (1963). Archives of Internal Medicine, 112,731 .

Artenstein, M. S., et al. (1971). Fournal of Infectious Diseases, 124, 277.

Barr, J., and Danielsson, D. (1971). British Medical fournal, 1, 482.

Benoit, F. L. (1963). American Fournal of Medicine, 35, 103.

Boger, W. P. (1944). American fournal of the Medical Sciences, 208, 708.

Boyle, J. A., and Buchanan, W. W. (1971). Clinical Rheumatology. Oxford, Blackwell.

Cattell, J. P. (1943). New England fournal of Medicine, 229, 49. $108,591$.

Fox, M. J., and Gilbert, J. (1944). American fournal of the Medical Sciences,

Greenwood, B. M., Whittle, H. C., and Dominic-Rajkovic, O. (1971). Lancet, 2, 519.

Herrick, W. W. (1918). Medical Clinics of North America, 2, 411.

Herrick, $W . W$. ., and Parkhurst, G. M. (1919). American fournal of the Medical Sciences, 158, 473

Hill, $\mathbb{W}$. R., and Kinney, T. D. (1947). Fournal of the American Medical Association, 134, 513.

Keefer, C. S., Parker, F., and Myers, W. K. (1934). Archives of Pathology, 18, 199.

Lapeyssonie, L. (1963). Bulletin of the World Health Organization, 28, Suppl. orse, J. R., Oretsky, M. I., and Hudson, J. A. (1971). Annals of Internal Medicine, 74, 212.

Ognibene, A. J., and Dito, W. R. (1964). Archives of Internal Medicine, 114,

Pinals, R. S., and Ropes, M. W. (1964). Arthritis and Rheumatism, 7, 241.

Silverstein, A. M., Welter, S., and Zimmerman, L. E. (1961). Fournal of Immunology, 86, 312 .

Waksman, B. H., and Bullington, S. J. (1956). Fournal of Immunology, 76, 441 .

\title{
Allergic Complications of Meningococcal Disease II-Immunological Investigations
}

\author{
B. M. GREENWOOD, H. C. WHITTLE, A. D. M. BRYCESON
}

British Medi cal fournal, 1973, 2, 737-740

\section{Summary}

Immunological investigation of four patients with meningococcal meningitis who developed arthritis or cutaneous lesions showed circulating meningococcal antigen at the time of presentation in each patient. It was cleared from the circulation over the next few days.

Department of Medicine, Ahmadu Bello University, Zaria, Nigeria B. M. GREENWOOD, M.D., M.R.C.P., Senior Lecturer H. C. WHITTLE, B.SC., M.R.C.P., Lecturer

A. D. M. BRYCESON, M.D., P.R.C.P., Senior Lecturer
Circulating antibody was detectable in three of the four patients about a week after the onset of the illness. A marked fall in the serum $C_{3}$ level occurred in two patients at about that time. Deposits of meningococcal antigen, immunoglobulin, and $C_{3}$ were detected in the synovial fluid white cells of the two patients studied and in one of three skin biopsies examined. These findings suggest that the arthritis and cutaneous lesions of meningococcal meningitis may be due to immune complex formation.

\section{Introduction}

Our previous paper showed how a proportion of patients with meningococcal meningitis developed arthritis and unusual 
vascular lesions during the period of recovery from their infection (Whittle et al., 1973). Bacteriological studies failed to implicate tissue invasion by active meningococci as the cause of these lesions. These complications appear most frequently about a week after the onset of the illness, a time at which the meningeal signs have usually resolved, suggesting that their pathogenesis may be related to the immune response of the host to the infection. The finding that the serum of some patients with meningococcal meningitis contains a circulating bacterial antigen (Edwards, 1971; Greenwood et al., 1971) and the fact that patients with circulating antigen are more likely to develop arthritis than those without (Greenwood et al., 1971) suggest that these lesions may be due to the formation of immune complexes. In this paper we describe detailed studies on four patients with meningococcal meningitis who developed arthritis or cutaneous lesions and present preliminary evidence which suggests that these lesions are due to immune complex formation.

\section{Materials and Methods}

Cerebrospinal fluid (C.S.F.) and sera from all patients with meningococcal meningitis admitted to Ahmadu Bello University Teaching Hospital, Zaria, during the period February to May 1972 were examined for the presence of meningococcal antigen. Repeated blood samples were obtained whenever possible from all patients who were antigen-positive on initial testing. Four of these patients subsequently developed arthritis or cutaneous lesions.

Meningococcal antigen was detected by counter-current immunoelectrophoresis as previously described (Greenwood et al., 1971). Antigen titres were determined by counter-current immunoelectrophoresis on doubling dilutions of serum.

Antibody levels to group A meningococcal antigen were determined by a haemagglutination method (Edwards and Driscoll, 1967). Previous experiments (B. M. Greenwood and H. C. Whittle, unpublished) have shown that the antigen used in this haemagglutination test gives a reaction of identity in precipitation tests with the antigen present in the C.S.F. and sera of some patients with meningococcal meningitis.

Serum $\mathrm{C}_{3}$ levels were measured by radial immunodiffusion and the results expressed as a percentage of a pool of normal European serum. Repeated estimations carried out on a control serum gave an accuracy of $\pm 10 \%$. The mean serum $\mathrm{C}_{3}$ level of 40 healthy Nigerian adults was $130 \% \pm 29 \%$ of the European serum standard.

Serial sera from two patients were tested for their ability to inhibit agglutination of human gammaglobulin-coated latex particles by a serum containing rheumatoid factor. The rheumatoid-factor-containing serum was used at a dilution of one-half the minimal agglutinating dose.

Selected sera were fractionated on Sephadex G 150 and the fractions obtained tested for immune complexes as described below.

Immunofluorescence was carried out on synovial fluid white cell preparations in the following way. Fresh samples were washed three times in medium 199 containing $5 \%$ inactivated calf serum and made up to a solution containing 1 million cells/ $\mathrm{mm}^{3}$. One drop of this suspension was added to $0.5 \mathrm{ml}$ of medium and spun in a cytocentrifuge for five minutes at 1,000 r.p.m. Cell preparations were fan dried and stained without washing or fixation. Skin and synovial biopsy specimens were taken as soon as lesions were noted. In no case were lesions more than 24 hours old when specimens were taken. Biopsy specimens were snap frozen with carbon dioxide. Six-micron sections were cut and washed in phosphate-buffered saline for 60 minutes before staining. The following sera were used in indirect and, when conjugated with fluorescein isothiocyanate, direct immunofluorescence: swine antihuman $\mathrm{IgA}, \mathrm{IgG}$, and IgM (Nordic Pharmaceuticals Ltd.); goat antihuman $C_{s}$ (kindly provided by Professor J. L. Turk); rabbit anti-group
A meningococcus (Burroughs Wellcome); and two sera raised by intravenous injection of rabbits with the Zaria strain of group A meningococci. None of the anti-meningococcal sera reacted with human gammaglobulin.

\section{CASE REPORTS}

Case 1.-A 17-year-old housewife was admitted to hospital on 12 February 1972 with a two-day history of fever and headache. She was stuporous and had numerous petechiae on the skin, palate, and conjunctivae. A skin petechia specimen was taken for biopsy. Purulent C.S.F. was obtained which grew Neisseria meningitidis. Treatment with crystalline penicillin 3 megaunits six-hourly was started and continued for the next five days. There was rapid improvement of fever, level of consciousness, and neck stiffness. On day 6 of her illness she developed a new skin lesion above the right elbow. A biopsy of the lesion was taken and specimens were examined by light and fluorescent microscopy. Two days later she developed episcleritis and an effusion in the right knee. The appearance of these new signs was associated with a secondary rise in fever. Fluid was aspirated from the right knee and a synovial biopsy was performed. The synovial fluid contained 57,000 white cells per $\mathrm{mm}^{3}$, of which $90 \%$ were polymorphonuclear neutrophils, $9 \%$ large mononuclear cells, and $1 \%$ lymphocytes. Organisms were not seen with Gram's stain and culture was sterile: Next day (day 9) she developed an effusion in the left knee, painful limitation of movement at the right wrist, and swelling around the right ankle. She was treated symptomatically with salicylates and the joint signs gradually resolved. Two weeks after presentation no abnormal physical signs were found.

Case 2.-A 40-year-old farmer was admitted to hospital on 21 February 1972 with headache and fever for one day. He was confused and febrile, had a stiff neck, and petechiae were present in both conjunctivae. Turbid C.S.F. was obtained from which $N$. meningitidis was isolated. Treatment with chloramphenicol $750 \mathrm{mg}$ six-hourly was started and continued for the next five days. He initially responded well to treatment but on day 7 of the illness he developed a triangular, macular lesion on his right arm about $1.5 \mathrm{~cm}$ in diameter with a dark centre and a blistered edge. A biopsy of the edge of the lesion was taken and specimens were subjected to light and fluorescent microscopy. Next day a crop of small nodular lesions developed around the initial lesion. No joint abnormalities were found. Thereafter he made a steady recovery and two weeks after the onset of his illness the skin lesions had nearly resolved.

Case 3.-An 8-year-old girl was admitted to hospital on 29 February 1972 with a three-day history of headache, fever, neck stiffness, and joint pains. She was febrile and confused and had a stiff neck. A few petechiae were seen in the conjunctiva of the left lower eyelid. She had generalized bone and joint tenderness but no definite signs of arthritis were found. Gram staining and culture of the C.S.F. were negative but group A meningococcal antigen was found in both C.S.F. and serum on counter-current immunoelectrophoresis. Treatment with chloramphenicol $500 \mathrm{mg}$ six-hourly was instituted and continued for the next five days. Her meningeal signs responded well to treatment but she continued to complain of generalized joint pains. Nevertheless, no objective signs of arthritis were found until day 8 of her illness, when a small effusion was noted in her left knee. This was aspirated and the fluid found to contain 54,000 white cells per $\mathrm{mm}^{3}$, of which $94 \%$ were polymorphs, $3 \%$ large mononuclear cells, and $3 \%$ lymphocytes. Her joint symptoms and signs gradually resolved over the next few days and when examined 17 days after the onset of the illness no abnormal physical signs were found.

Case 4.-A $1 \frac{1}{2}$-year-old boy was admitted to hospital on 23 February 1972 with a two-day history of fever, neck stiffness, and convulsions. He was febrile and semiconscious. There was no neck stiffness but petechiae were present on the conjunctivae and palate. C.S.F. was turbid and $N$. meningitidis was isolated on culture. Treatment with crystalline penicillin 1 megaunit six-hourly was started and continued for the next five days. He responded well until the ninth day of his illness, when he developed a bullous eruption on the medial aspect of the right knee associated with a secondary rise in fever. A biopsy of this lesion was taken and specimens were examined by light and fluorescent microscopy. On day 12 of his illness an effusion was noted in the left knee. Thereafter no new lesions developed and he was discharged two days later. He failed to attend for further follow-up and could not be traced. 


\section{Results}

SERUM

The results of serial estimations of group A meningococcal antigen, anti-meningococcal antibody, and $\mathrm{C}_{3}$ levels in the four patients are shown in the table. In each patient circulating

Serial Estimations of Antigen ( $A g)$, Antibody $(A b)$, and $C_{3}$ in Cases 1 to 4 Antigen and Antibody Levels are expressed as Reciprocal of Titre in Doubling Dilutions; $C_{3}$ Levels are expressed as Percentage of Pool of Normal European Sera

\begin{tabular}{|c|c|c|c|c|c|c|c|c|c|c|c|c|}
\hline \multirow{2}{*}{$\begin{array}{l}\text { Day of } \\
\text { Illness }\end{array}$} & \multicolumn{3}{|c|}{ Case 1} & \multicolumn{3}{|c|}{ Case 2} & \multicolumn{3}{|c|}{ Case 3} & \multicolumn{3}{|c|}{ Case 4} \\
\hline & $\mathbf{A g}$ & $\mathrm{Ab}$ & $\mathrm{C}_{8}$ & $\mathbf{A g}$ & $\mathrm{Ab}$ & $\mathrm{C}_{3}$ & Ag & $\mathrm{Ab}$ & $\mathrm{C}_{3}$ & Ag & $\overline{A b}$ & $\mathrm{C}_{3}$ \\
\hline $\begin{array}{r}1 \\
2 \\
3 \\
4 \\
5 \\
6 \\
7 \\
8 \\
9 \\
10 \\
12 \\
14 \\
16 \\
18\end{array}$ & $\begin{array}{l}\mathbf{4} \\
\mathbf{2} \\
\mathbf{2} \\
- \\
= \\
= \\
= \\
= \\
- \\
-\end{array}$ & $\begin{array}{l}- \\
- \\
\overline{1} \\
2 \\
2 \\
4 \\
3 \\
3 \\
4 \\
2\end{array}$ & $\begin{array}{r}84 \\
127 \\
150 \\
88 \\
112 \\
127 \\
168 \\
149 \\
160 \\
180 \\
168\end{array}$ & $\begin{array}{l}1 \\
2 \\
2 \\
2 \\
- \\
- \\
- \\
-\end{array}$ & $\begin{array}{l}\bar{z} \\
\bar{z} \\
\bar{z} \\
1 \\
2 \\
1\end{array}$ & $\begin{array}{r}70 \\
76 \\
84 \\
88 \\
44 \\
84 \\
96 \\
121\end{array}$ & 2 & $\overline{3}$ & $\begin{array}{l}132 \\
180 \\
116 \\
200\end{array}$ & $\begin{array}{r}3 \\
3 \\
3 \\
-\end{array}$ & $\begin{array}{l}- \\
\overline{-} \\
1\end{array}$ & $\begin{array}{r}55 \\
62 \\
75 \\
64 \\
\\
116\end{array}$ \\
\hline
\end{tabular}

antigen was present at the time of presentation and this disappeared over the next one to four days. Circulating antibody was first detectable at about the fifth day of the illness. $\mathrm{C}_{3}$ levels were low at the time of presentation in three of the patients and rose gradually over the next few days. A marked drop then occurred in the serum $C_{3}$ level of cases 1 and 2 (fig. 1), this fall

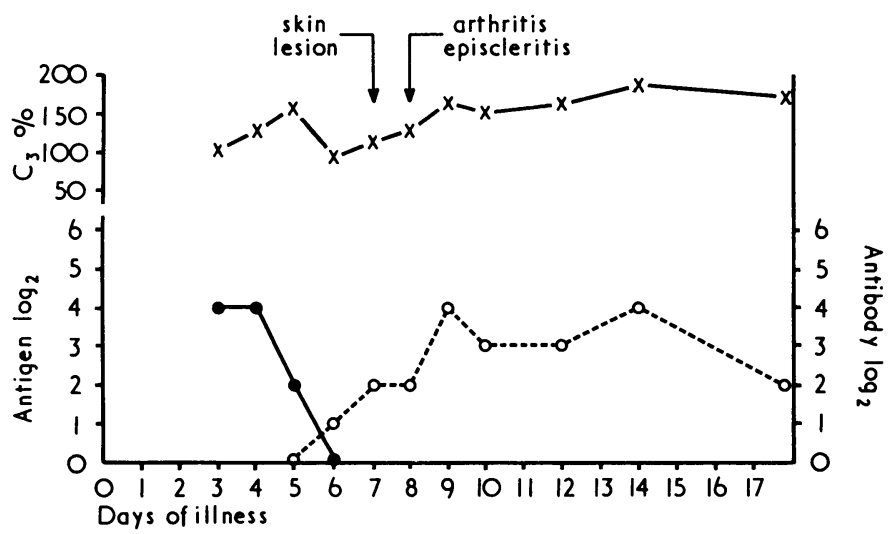
FIG. 1 -Serial estimations of antigen $(-\triangle)$, antibody $(0-0)$, and arthritis and cutaneous vascular lesions.

coinciding with the disappearance of antigen from the circulation. Allergic complications were first noted in these two patients the day after the fall in $\mathrm{C}_{3}$ level.

Sera taken at the time that cutaneous lesions or arthritis were first detected were tested for immune complexes. After fractionation on Sephadex G 150 the macroglobulin peak was dialysed against glycine saline buffer, $\mathrm{pH} 3 \cdot 0$, in an attempt to split complexes and the solution tested for the presence of meningococcal antigen by counter-current immunoelectrophoresis. In no case was antigen shown. Protein was precipitated from this solution by addition of an equal volume of $10 \%$ trichloroacetic acid, the supernatant dialysed against phosphatebuffered saline at $\mathrm{pH} 7 \cdot 2$, and tested for meningococcal antigen again by counter-current immunoelectrophoresis. No antigen could be found.
Serial sera from cases 1 and 2 were tested for their ability to inhibit agglutination of human gammaglobulin-coated latex particles by rheumatoid factor. Inhibition at a titre of $1 / 10$ was given by several of the serial samples from case 2 but no increase in titre was found at the time that the patient developed allergic complications.

\section{SYNOVIAL FLUID}

Cell preparations were made from synovial fluid obtained from cases 1 and 3. Globular deposits staining for $\operatorname{IgM}, \operatorname{IgG}, C_{3}$, and meningococcal antigen were shown by direct and indirect immunofluorescence in cells from case 1 (fig. 2). The deposits

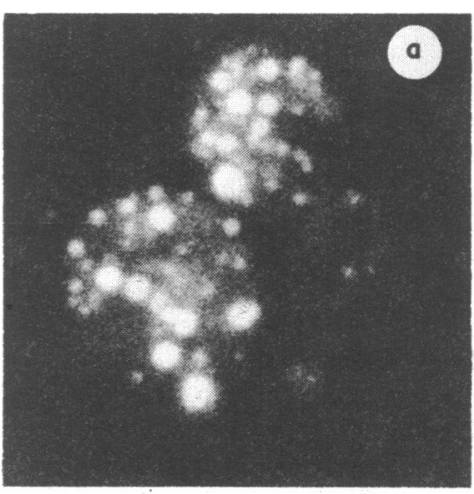

FIG. 2-Case 1. (a) Synovia fluid white cells, and Synovia biopsy specimen of lesion taken biopsy specimen of lesion taken on day 6 stained by direct immunofluorescence for meningococcal antigen. Similar pattern of staining were given by anti$\operatorname{Ig} M$ and anti- $\mathrm{C}_{3}$ fluorescent conjugates.

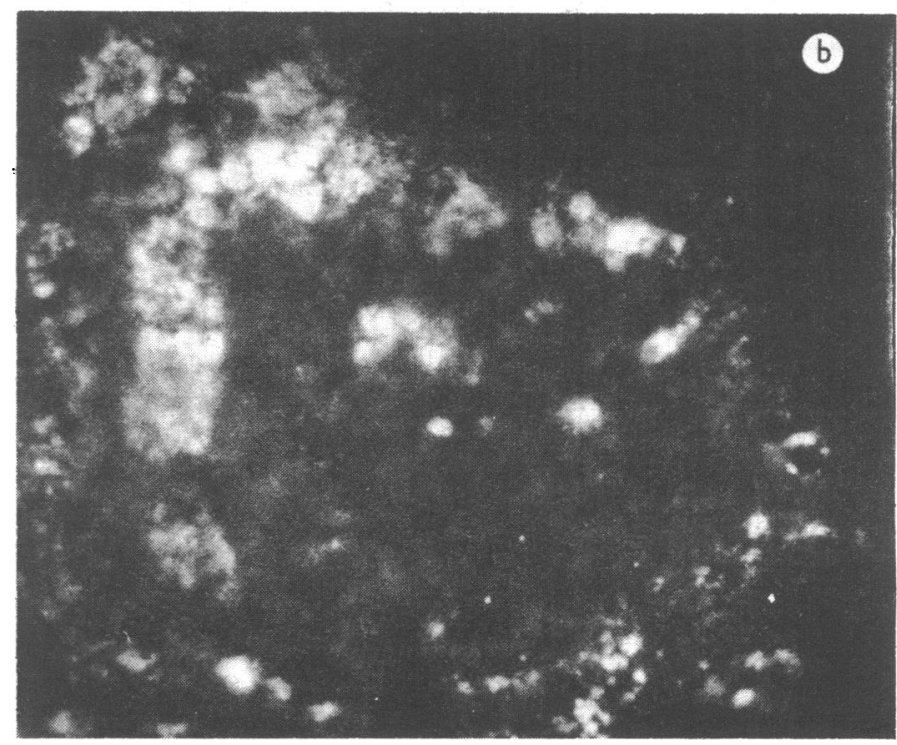

were about $2 \mu \mathrm{m}$ in diameter and were present in both polymorphs and mononuclear cells. Anti-IgM conjugates gave brighter staining than anti-IgG conjugates. The specificity of the meningococcal staining was shown by the fact that the staining could be almost completely abolished by absorbing the antimeningococcal conjugate with purified meningococcal antigen.

Synovial fluid cells from case 3 contained similar globular deposits staining for $\operatorname{IgM}, \mathrm{C}_{3}$, and meningococcal antigen but the proportion of cells showing staining was less than that observed in case 1 .

Synovial fluid cells from a patient with Reiter's syndrome and from a patient with pneumococcal arthritis contained no globular deposits when stained with the conjugates described above. 


\section{SYNOVIAL MEMBRANE}

Light microscopy of the synovium obtained from case 1 showed oedema and infiltration with polymorphs and large mononuclear cells.

On fluorescent microscopy two areas of fluorescence were observed about $100-200 \mu \mathrm{m}$ in diameter. These areas stained strongly for IgM, IgG, and meningococcal antigen and weakly for $C_{3}$. A small number of cells containing globular deposits similar to those observed in synovial fluid cells were also observed.

\section{SKIN}

Case 1.-Histological examination of the early (day 3) petechial lesion showed oedema of the epidermis, dislocation of pigment, and infiltration of the basal layer with polymorphs. The dermis showed oedema and haemorrhage and contained congested vessels. Direct immunofluorescence with antimeningococcal and anti- $\mathrm{C}_{3}$ conjugates showed speckled intracellular staining within and around dermal vessels. This pattern was not observed using anti-immunoglobulin antisera nor was it observed in normal African skin. The late (day 6) lesion showed a mild perivascular infiltration with mononuclear cells of the small dermal vessels, some of which were congested and oedematous. Direct immunofluorescence showed chunky deposits in and around the wall of a large, deep dermal vessel that stained with anti-IgM, anti- $\mathrm{C}_{3}$, and anti-meningococcal conjugates but not with an anti-IgG conjugate (fig. 2). Two smaller vessels showed similar but less intense staining. In addition the area of cellular exudate around some small vessels stained with an anti-IgG conjugate and weakly with anti-IgM and anti- $\mathrm{C}_{3}$ conjugates. On indirect immunofluorescence with an anti-meningococcal serum a similar pattern of staining was observed. The chunky deposits were not stained by anti-albumin, anti-transferrin, or normal rabbit sera.

Case 2.-Histologically there was atrophy of the epidermis and infiltration of the basal layer with polymorphs. The dermis was oedematous and infiltrated with mononuclear cells. Small vessels were congested and infiltrated with polymorphs and mononuclear cells. Some of the smaller vessels were thrombosed. One artery was grossly oedematous with necrosis of the intima and infiltration of all coats. Immunofluorescent staining failed to show any deposits of immunoglobulin, meningococcal antigen, or $\mathbf{C}_{3}$.

Case 4.- Histological examination showed congestion of small, superficial dermal vessels which contained an excess of mononuclear cells. One deep artery was thrombosed and its walls were infiltrated with polymorphs. There was also marked perivascular cuffing with polymorphs and mononuclear cells. Immunofluorescent staining failed to show deposits of immunoglobulin, $\mathrm{C}_{3}$, or meningococcal antigen.

\section{Discussion}

The results of this study support the view that the arthritis and cutaneous lesions of patients recovering from meningococcal meningitis are related to the immune response of the host rather than to direct invasion of the tissues by the organism. Serological studies in the four patients showed a fall in the titre of circulating meningococcal antigen during the first few days of the illness, followed in three patients by a rise in antibody level to the same antigen. A suitable situation for immune complex formation might therefore have occurred in these patients about one week after the onset of their illness. A fall in the serum $\mathrm{C}_{3}$ level of two and possibly three patients suggests that this may have occurred.

Globular deposits of meningococcal antigen, meningococcal antibody, and $\mathrm{C}_{3}$ were found in the synovial fluid cells of the two patients investigated. It was not established that antigen, antibody, and $\mathrm{C}_{3}$ were present in the same cells but the similar pattern of staining obtained with different conjugates makes this likely. Deposits of antigen, antibody, and $\mathrm{C}_{3}$ were, however, present in the same areas of serial sections of skin and synovium, suggesting the presence of complexes. Complexes were found in only one of the three skin biopsy specimens examined. Histological findings of the skin of the two negative patients, however, were consistent with a complex-induced vasculitis. Experimental studies (Cream et al., 1971) have shown that the development of an inflammatory response soon makes it difficult to demonstrate the presence of complexes known to have been deposited in the skin. Though all our biopsy samples were taken within 24 hours of the appearance of a skin lesion this may still have been too late to allow the demonstration of complexes by immunofluorescence. Alternatively the sections examined may have missed the vessel primarily affected and shown only subsequent surrounding inflammatory changes.

The presence of complexes in the skin and synovium of patients with meningococcal meningitis could be due to the formation and subsequent deposition of circulating immune complexes or to an Arthus phenomenon. Skin and synovium are favoured sites for the deposition of circulating immune complexes, and deposition of circulating complexes could account for the episcleritis observed in case 1. Glomerulonephritis, however, does not appear to be a frequent complication of meningococcal meningitis (Whittle et al., 1973) though the renal glomerulus is a favoured site for immune complex deposition. Direct attempts to demonstrate the presence of circulating immune complexes were unsuccessful but this failure may have been due to technical difficulties, and further attempts to demonstrate the presence of circulating complexes are in progress.

The circulating meningococcal antigen present in each patient on admission disappeared from the circulation over the next few days and was probably taken up by tissue macrophages. Bacterial polysaccharides are very resistant to digestion and may persist within phagocytic cells for long periods. Persistence of antigen in the phagocytic cells of the skin and the synovium until the time that the patient formed circulating antibody would create a suitable situation for the development of an Arthus reaction at these sites, and this may have occurred in our patients. At present our data are inadequate to differentiate between these two possible mechanisms for the production of arthritis and vascular lesions in patients with meningococcal meningitis.

Our conclusion that the arthritis and cutaneous lesions of patients recovering from meningococcal meningitis are related to the immune reaction of the host is of importance in the clinical management of patients with this disease, for prolonged antibiotic therapy is unlikely to be of any value in the treatment of these complications. Detection of circulating antigen by counter-current immunoelectrophoresis at the time of presentation helps to identify patients especially at risk of developing these complications. As these complications can be severe (Whittle et al., 1973) the question of whether this high-risk group of patients might benefit from early treatment with corticosteroids is being considered.

We wish to thank Miss J. C. Boler for her skilled technical help. This study was supported by grants from the Foreign and Commonwealth Office (Overseas Development Administration) and the Medical Research Council.

\section{References}

Cream, J. J., Bryceson, A. D. M., and Ryder, G. (1971). British fournal of Dermatology, 84, 106.

Edwards, E. A. (1971). Fournal of Immunology, 106, 314.

Edwards, E. A., and Driscoll, W. S. (1967). Proceedings of the Society of Experimental Biology and Medicine, 126, 876.

Greenwood, B. M., Whittle, H. C., and Dominic-Rajkovic, O. (1971). Lancet, $2,519$.

Whittle, H. C., et al. (1973). British Medical fournal, 1973, 2, 733. 